\title{
Evidence for a causal relationship between the structure, size, and load of calcium pyrophosphate dihydrate crystals, and attacks of pseudogout
}

\author{
Angela Swan, Brigid Heywood, Barry Chapman, Heather Seward, Paul Dieppe
}

\begin{abstract}
Objective-To investigate any relationship between the nature, size, and numbers of synovial fluid (SF) calcium pyrophosphate dihydrate (CPPD) crystals, and attacks of pseudogout.

Methods-Knee SF was aspirated from nine selected patients, first during an attack of pseudogout (acute sample) and again later when the attack had subsided (interval sample). CPPD crystals were extracted, weighed, examined by high resolution transmission electron microscopy (HRTEM), and characterised by size and crystal habit (monoclinic or triclinic). Structural analysis was carried out by $x$ ray powder diffraction (XRD) and the proportions of monoclinic to triclinic CPPD were estimated from densitometric measurements of selected key reflections. Results-The mean crystal size, by HRTEM, indicated that the crystals in the acute sample were larger than those in the interval sample. The ratio of monoclinic to triclinic CPPD, whether estimated from their morphological appearance by HRTEM, or from XRD, was greater in the acute than in the interval sample in all nine patients. The total amount of extracted mineral varied, but in every patient the concentration of CPPD per $\mathrm{ml}$ of fluid, and the total mineral per joint, were greater in the acute sample than in the interval sample.
\end{abstract}

Conclusion-In this highly selected group of patients, the large numbers of CPPD crystals associated with attacks of pseudogout included a greater proportion of monoclinic crystals, and larger crystals, than those present when inflammation had subsided. A special, phlogistic population of crystals may exist, originating in different joint tissues, or cleared in a different manner, than the more common populations of smaller crystals with a greater proportion of triclinic CPPD, seen in chronic disease.

(Ann Rheum Dis 1995; 54: 825-830)

The deposition of calcium pyrophosphate dihydrate (CPPD) crystals in joints is a common, age-related phenomenon. ${ }^{1}$ Large deposits can be detected radiographically (chondrocalcinosis (CCA)) and are particularly common in the knee joint, affecting both fibrocartilage menisci and hyaline articular cartilage. ${ }^{2}$ Crystallographic analysis of these deposits has invariably shown them to contain mixtures of monoclinic and triclinic CPPD crystals of varying sizes. ${ }^{3}$ People with radiographic evidence of CPPD deposits in their knee joints may have no evidence of joint disease; however, there are two well established clinical associations: attacks of acute, self limiting synovitis (pseudogout), and osteoarthritis (OA). ${ }^{45}$ Both in patients with pseudogout and in patients with OA and CCA, CPPD crystals are generally present in the synovial fluid (SF). Several investigators have shown that CPPD crystals can cause inflammation ${ }^{6} 7$ and it is widely accepted that these crystals cause the inflammation of pseudogout. However, one of the enigmas of the CPPD related disorders, as with other forms of crystal arthropathy, is the fact that the crystals appear to cause attacks of severe synovitis, but are also frequently found free in the joint fluid between attacks, and in patients with OA in whom there is very little synovitis.

There are several possible explanations for this enigma. The crystal load in the joint may be critical, and it has been reported previously that fluids from patients with pseudogout tend to contain more CPPD than those from OA patients. ${ }^{8}$ Alternatively, either the size or structure of the crystals might be important. The size of CPPD crystals identified in joint tissues and fluids varies considerably, and they are of two types, either monoclinic or triclinic in lattice structure. Using the right hand screw convention for the labelling of axes and interaxial angles of crystals, in the monoclinic system the axial restriction is that $\alpha=\beta=90$ degrees; in the triclinic system there are no symmetry elements. For CPPD, the usual visual expressions of these symmetries (crystal habits) are elongated rod-like for monoclinic $(\mathrm{M}-) \mathrm{CPPD}$, and 'squat' rhomboid or cuboid for triclinic ( $T$-)CPPD. It follows from mathematical principles that the surface area:volume ratio of the longer, rod-like $M-C P P D$ is greater than that of T-CPPD. There is some evidence in the literature that smaller crystals can be more phlogistic than larger ones, ${ }^{9}$ that the structure of crystals, in addition to their surface identity, is important in determining inflammatory response, ${ }^{10}$ and that monoclinic CPPD may be more active than the triclinic form. ${ }^{11}$ Finally, the protein coating of the crystal might be the dominant factor. ${ }^{12} 13$ 
In order to examine this, we have studied the synovial fluid crystal load and the size and crystallographic identity of the individual crystals in patients with pseudogout, both during an attack, and at a time in which there was little or no evidence of synovitis.

\section{Patients and methods PATIENTS}

Patients presenting with pseudogout of the knee joint were included in this study. Each patient presented with the sudden onset of pain and swelling of the knee joint, radiographic evidence of CCA and a synovial fluid aspirate which had a high count of polymorphonuclear leucocyte (PMN) cells, and CPPD crystals on polarised light microscopy. The patients were treated by aspiration of as much synovial fluid as possible from the knee, followed by injection of a long acting intra-articular steroid preparation. The patients were followed up over the next six months, and in those patients in whom the affected joint became cool and painless, but showed clinical evidence of an effusion, a second SF sample ('interval' sample) was obtained, the operator again attempting to aspirate as much fluid as possible from the joint. Sequential SF samples suitable for the extraction and analysis of crystals were obtained from nine subjects. In each case, knee radiographs were obtained during the six months of the study and examined by a single observer who recorded the presence of CCA and graded it as minor (1), moderate (2) or severe (3) in amount, in addition to recording the grade of OA on the Kellgren and Lawrence scale. ${ }^{14}$

\section{SYNOVIAL FLUID EXAMINATION AND}

TREATMENT

The synovial fluids were aspirated via a 21 gauge $(0.8 \times 40 \mathrm{~mm})$ needle, into syringes which were immediately capped; SF were examined, and usually processed within two hours of joint aspiration. When crystal extraction could not be carried out immediately, the SF samples were stored uncentrifuged, in sterile tubes with minimal air space, at $-70^{\circ} \mathrm{C}$ for up to two weeks before processing; it has been shown that storage under these conditions, for this time, does not significantly alter the CPPD content of SF samples. ${ }^{15}$

The total fluid volume was measured and a small aliquot examined for total leucocyte count using a haemocytometer; a differential leucocyte count was performed on an air dried smear. The presence of CPPD-like crystals was confirmed by polarised light microscopy using a Zeiss Axioplan microscope, incorporating a polarised light facility, with first order red quartz plate. The remainder of the sample was processed for extraction of mineral as previously described and validated. ${ }^{16}$ Briefly, the fluids were incubated at $37^{\circ} \mathrm{C}$ with papain $1 \mathrm{mg} / \mathrm{ml} \mathrm{SF} \mathrm{w/v} \mathrm{(Sigma} \mathrm{P4762),} \mathrm{then} \mathrm{with}$ fresh sodium hypochlorite solution (final concentration $0.5 \% \mathrm{v} / \mathrm{v})$. The extracted deposit was washed five times in sterile deionised dis- tilled water, and finally washed in $98 \% \mathrm{v} / \mathrm{v}$ ethanol and dried at $37^{\circ} \mathrm{C}$. The small pellet of mineral deposit was then weighed on a Cahn 21 microbalance, before microscopic and analytical examination.

EXAMINATION OF THE EXTRACTED CRYSTALS Samples of the extracted crystal pellets were examined by polarised light microscopy, high resolution transmission electron microscopy (HRTEM) and $x$ ray diffraction (XRD).

HRTEM was carried out using a JEOL 200CX high resolution transmission electron microscope, operating at $100 \mathrm{KV}$, and crystals were examined under conditions of minimal illumination. For statistical analysis, the crystals were characterised on the basis of size, and structure (monoclinic and triclinic); the population minimum was 100 crystals and measurements were taken from at least three randomly selected micrographs of each sample.

Structural analysis by XRD used a PW 1010/80 Phillips $x$ ray generator: $\mathrm{Cu} \mathrm{K} \alpha$ radiation plus nickel filter, with a Debye-Scherrer powder camera $(114.83 \mathrm{~mm}$ diameter). The relative intensity of the XRD powder lines in any one film was measured by means of a Joyce-Loebl double beam recording microdensitometer, Mk 111C. Principal XRD reflections were used to distinguish between the two morphological forms of CPPD; these were at d-spacing $8.04 \AA$ (intensity $\mathrm{I} / \mathrm{I} 1100$ ) (reflection A) for T-CPPD, and at d-spacing $7.37 \AA$ (intensity $\mathrm{I} / \mathrm{I} 1 \mathrm{100}$ ) (reflection B) and $4.65 \AA$ (intensity $\mathrm{I} / \mathrm{I} 1$ 90) (reflection $\mathrm{C}$ ) for $M-C P P D$. Because the small quantities of biogenic material available restricted direct quantitative analysis of the composition by $\mathrm{XRD}$, a compromise method was based on densitometric measurements of peak height of the selected reflections (peaks A, B, and C). From these data, estimates of the relative proportion of M-CPPD and T-CPPD could be determined for each sample, with variations in this ratio indicating changes in the crystal population over time.

\section{Results}

Table 1 shows details of the nine patients. There were five women and four men (mean age 75.4 (range 55-86) years). Seven had evidence of $\mathrm{OA}$, in addition to CCA on the knee radiograph. The interval between collection of the fluid during an acute attack and aspiration of the second (interval) sample varied from four to 24 weeks (mean interval 10 weeks). The volume of SF obtained varied from 3 to $50 \mathrm{ml}$, and the leucocyte count from $0 \cdot 1$ to $160 \times 10^{5} / \mathrm{ml} \mathrm{SF}$, but in each of the patients both the total volume of fluid aspirated and the PMN cell count were greater in the fluid obtained during the acute attack than in the interval sample.

All nine patients had radiological evidence of CCA; there was no apparent relationship between the amount of CCA or the amount of OA change seen on radiographs, and any of the 
Table 1 Patient and synovial fluid $(S F)$ data

\begin{tabular}{|c|c|c|c|c|c|c|c|c|}
\hline \multirow[t]{3}{*}{ Patient } & \multirow{3}{*}{$\begin{array}{l}\text { Age } \\
(y r)\end{array}$} & \multirow[t]{3}{*}{ Sex } & \multicolumn{2}{|l|}{$x$ Ray } & \multicolumn{2}{|c|}{ SF volume (ml) } & \multirow{2}{*}{\multicolumn{2}{|c|}{$\begin{array}{l}\text { Leucocyte count } \\
\left(\times 10^{5}\right)\end{array}$}} \\
\hline & & & \multirow{2}{*}{$\begin{array}{l}\text { CCA } \\
\text { grade }\end{array}$} & \multirow{2}{*}{$\begin{array}{l}K \mathcal{E} L \\
\text { score }\end{array}$} & \multirow{2}{*}{ Acute } & \multirow{2}{*}{ Interval } & & \\
\hline & & & & & & & Acute & Interval \\
\hline 1 & 81 & $\mathbf{M}$ & 2 & 0 & 30 & 15 & 10 & 0.1 \\
\hline 2 & 86 & $\mathbf{M}$ & 2 & 3 & 25 & 9 & 20 & 5 \\
\hline 3 & 78 & $\mathbf{F}$ & 1 & 3 & 5 & 5 & .10 & 1 \\
\hline 4 & 80 & $\mathbf{F}$ & 2 & 4 & 5 & 4 & 10 & 0.1 \\
\hline 5 & 55 & $\mathbf{F}$ & 2 & 2 & 50 & 6 & 20 & 0.1 \\
\hline 6 & 75 & $\mathbf{F}$ & 1 & 3 & 20 & 6 & 20 & 2 \\
\hline 7 & 69 & $\mathbf{M}$ & 1 & 0 & 15 & 5 & 27 & $<1$ \\
\hline 8 & 72 & $M$ & 1 & 1 & 5 & 3 & 21 & $<1$ \\
\hline 9 & 83 & $\mathrm{~F}$ & 1 & 2 & 29 & 21 & 160 & 5 \\
\hline
\end{tabular}

CCA $=$ Chondrocalcinosis - grades: $0=$ none; $1=$ mild; $2=$ severe .

$\mathrm{K} \& \mathrm{~L}=\mathrm{Kellgren}$ and Lawrence score for $\mathrm{OA}(0=$ none $\ldots .4=$ severe $)$

SF findings (table 1). Table 2 shows the amount and nature of the mineral recovered from each SF sample, the crystal size data, and the ratio of monoclinic to triclinic crystals in each sample (by HRTEM and by densitometry of XRD principal reflections). The total amount of mineral varied widely, from 10 to $13000 \mu \mathrm{g}$, but in each individual there was considerably more mineral per millilitre of SF, and more total mineral per joint, in the sample obtained during an acute attack than that taken in the interval period. Table 2 shows that calcium hydroxyapatite (HAP) crystals were also detected in both acute and interval SF from four patients; in one patient HAP crystals were detected only in the acute sample; another patient had detectable HAP crystals only in the interval sample. In three patients, neither the acute nor the interval sample contained detectable HAP. Thus there was no pattern of association of SF HAP crystals with inflammation. The amount of HAP detected was invariably very small (just a few HAP crystals detected by HRTEM); there was no apparent reduction in HAP crystal numbers during interval episodes, but the very low concentrations made quantification impossible.

Additional information on approximate crystal surface area was calculated from measurements of aspect ratios of extracted biogenic M-CPPD and T-CPPD crystals from the electron micrographs. The results provided some evidence that the mean surface area of monoclinic CPPD (40.20 (SD 75.70) $\mu \mathrm{m}^{2}$; range $\left.0.94-283.30 \mu \mathrm{m}^{2}(\mathrm{n}=20)\right)$ was greater than that of triclinic CPPD $(27 \cdot 38(37 \cdot 60)$ $\mu \mathrm{m}^{2}$; range $\left.0 \cdot 78-165 \cdot 12 \mu \mathrm{m}^{2}(\mathrm{n}=20)\right)$. The results clearly showed the major contribution made to total available surface area by the larger crystals in this sample though, weight for weight, smaller crystals will always provide a greater specific surface area than large ones. Unfortunately, insufficient biogenic material was available for the far more accurate measurement of crystal surface area by gas absorption methods.

Electron micrographs of CPPD crystals extracted from SF from patient 6 illustrate the presence of larger, monoclinic CPPD in the acute sample (fig 1) compared with the interval sample (fig 2) which contains smaller, mainly triclinic CPPD. Figures 3 and 4 illustrate the XRD films of these two populations of extracted CPPD crystals from sample 6, together with the corresponding densitometric traces. The principal reflections $\mathrm{A}, \mathrm{B}$, and $\mathrm{C}$, from which the proportion of monoclinic to triclinic CPPD was calculated for all the samples, are indicated on the traces.

The estimates of mean crystal size, based on examination by HRTEM of a minimum of 100 representative crystals per sample, indicated that the crystals in the acute attack samples tended to be larger than those found in the interval fluids. In addition, the ratio of monoclinic to triclinic crystals, whether estimated from their morphological appearance by HRTEM, or by densitometry of XRD key reflections, showed a consistent pattern of a much greater proportion of monoclinic crystals in the acute than in the interval SF samples.

\section{Discussion}

The objective of this study was to examine the hypothesis that acute attacks of pseudogout are associated with a number and type of CPPD crystals in the SF different from those found in patients presenting with little or no synovitis. The hypothesis was based on two previous observations: the cross sectional data to indicate that more CPPD is found in SF from patients with pseudogout than in those with $\mathrm{OA}$ and chondrocalcinosis, ${ }^{8}$ and the suggestion that monoclinic CPPD might be more phlogistic than the triclinic form. ${ }^{11}$ In order to test the hypothesis, it is clearly desirable to examine sequential SF samples rather than cross sectional samples, because of the variation both in patients with CPPD related disorders and in their SF findings. Patients with pseudogout usually recover quickly from an acute attack of synovitis, particularly if treated with

Table 2 Extracted crystal data

\begin{tabular}{|c|c|c|c|c|c|c|c|c|c|c|c|c|}
\hline & \multirow{2}{*}{\multicolumn{4}{|c|}{$\begin{array}{l}\text { Concentration in } \\
\text { SF and total }\end{array}$}} & \multirow{2}{*}{\multicolumn{2}{|c|}{$\begin{array}{l}\text { HRTEM M-CPPD } \\
\text { mean length }(\mu \mathrm{m})\end{array}$}} & \multicolumn{6}{|c|}{$M: T C P P D$ ratio } \\
\hline & & & & & & & \multicolumn{2}{|c|}{ HRTEM } & \multicolumn{4}{|c|}{$X R D$} \\
\hline & \multicolumn{2}{|l|}{ Acute } & \multicolumn{2}{|l|}{ Interval } & \multirow[t]{2}{*}{ Acute } & \multirow[t]{2}{*}{ Interval } & \multirow{2}{*}{ Acute } & \multirow{2}{*}{ Interval } & \multicolumn{2}{|c|}{ Acute } & \multicolumn{2}{|c|}{ Interval } \\
\hline & $\begin{array}{l}\text { Concn } \\
(\mu g / m l)\end{array}$ & $\begin{array}{l}\text { Total } \\
(\mu g)\end{array}$ & $\begin{array}{l}\text { Concn } \\
(\mu g / m l)\end{array}$ & $\begin{array}{l}\text { Total } \\
(\mu g)\end{array}$ & & & & & $B: A$ & $C: A$ & $B: A$ & $C: A$ \\
\hline $\begin{array}{l}1 \\
2 \\
3 \\
4 \\
5 \\
6 \\
7 \\
8 \\
9 \\
9\end{array}$ & $\begin{array}{r}100 \\
392 \\
2600 \\
110 \\
40 \\
13 \\
84 \\
36 \\
110\end{array}$ & $\begin{array}{r}3000 \\
9800 \\
13000 \\
550 \\
2000 \\
234 \\
1260 \\
180 \\
3190\end{array}$ & $\begin{array}{r}22 \\
60 \\
2 \\
32 \\
10 \\
5 \\
12 \\
40 \\
13\end{array}$ & $\begin{array}{r}330 \\
540 \\
10 \\
128 \\
60 \\
30 \\
60 \\
120 \\
273\end{array}$ & $\begin{array}{l}3 \cdot 8^{\prime} \\
9 \cdot 2^{\star} \\
8 \cdot 4^{\star} \\
3 \cdot 8^{\star} \\
3 \cdot 9^{\star} \\
5 \cdot 6 \\
4 \cdot 6 \\
6 \cdot 4^{\star} \\
6 \cdot 4^{\star}\end{array}$ & $\begin{array}{l}2 \cdot 8 \\
4 \cdot 9^{\star} \\
5 \cdot 3^{\star} \\
2 \cdot 4^{\star} \\
2 \cdot 3^{\star} \\
3 \cdot 8^{\star} \\
3 \cdot 6^{\star} \\
3 \cdot 3^{-} \\
3 \cdot 6\end{array}$ & $\begin{array}{l}4 \cdot 0 \\
6 \cdot 2 \\
6 \cdot 0 \\
4 \cdot 3 \\
3 \cdot 6 \\
6 \cdot 0 \\
6 \cdot 6 \\
5 \cdot 2 \\
7 \cdot 0\end{array}$ & $\begin{array}{l}2 \cdot 0 \\
2 \cdot 4 \\
2 \cdot 0 \\
2 \cdot 7 \\
2 \cdot 1 \\
2 \cdot 7 \\
2 \cdot 2 \\
2 \cdot 4 \\
2 \cdot 4\end{array}$ & $\begin{array}{l}1.6 \\
0.9 \\
0.8 \\
1.5 \\
1.1 \\
8.0 \\
1.6 \\
1.6 \\
1.2\end{array}$ & $\begin{array}{l}0 \cdot 6 \\
0 \cdot 4 \\
0 \cdot 3 \\
1 \cdot 3 \\
0 \cdot 6 \\
3 \cdot 8 \\
0 \cdot 8 \\
1 \cdot 2 \\
0 \cdot 8\end{array}$ & $\begin{array}{l}1.0 \\
0.3 \\
\text { NA } \\
0.9 \\
0.5 \\
1.0 \\
0.6 \\
0.7 \\
0.3\end{array}$ & $\begin{array}{l}0.3 \\
0.2 \\
\text { NA } \\
0.4 \\
0.4 \\
0.5 \\
0.3 \\
0.5 \\
0.2\end{array}$ \\
\hline
\end{tabular}

*Small numbers of calcium hydroxyapatite crystals also detected by HRTEM. 


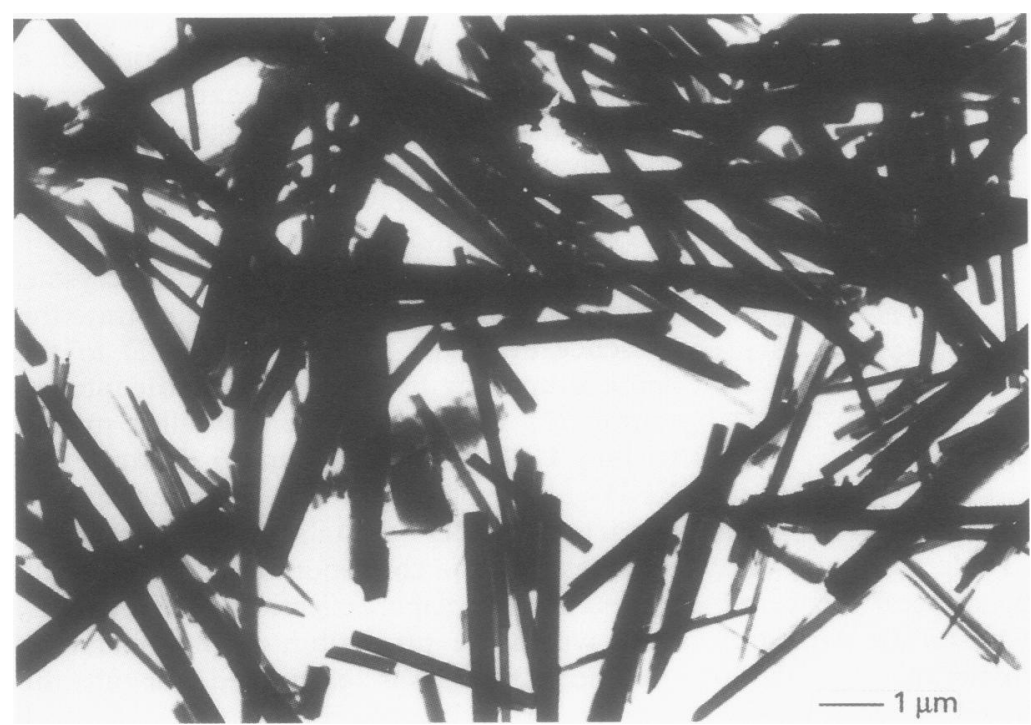

Figure 1 Electron micrograph of calcium pyrophosphate dihydrate crystals extracted from acute synovial fluid sample from patient 6 . Total yield of crystals $234 \mu \mathrm{g}$; almost entirely monoclinic (rod shaped), with a mean length of $5.6 \mu \mathrm{m}$.

intra-articular steroids, ${ }^{17}$ but may have persistent small effusions containing CPPD crystals between attacks. These features make these pseudogout patients an ideal group for investigation, and a group of nine patients with classical diagnoses of pseudogout (based upon clinical, radiographic, and SF findings) was selected for this sequential study. It should be noted, however, that this was a highly selected group, because many of those who were successfully treated for pseudogout had either no effusion to aspirate, or too little SF for mineral extraction at the follow up visits. It is also clear that the group selected for study did have some intra-articular inflammation at the time at which the interval sample was aspirated; they had, by definition, an effusion, and the SF analysis showed slightly greater leucocyte counts than are found in normal SF. ${ }^{18}$ In spite of these reservations, the strength of the study is that it allowed comparison of two sequential samples from each patient, and that in every

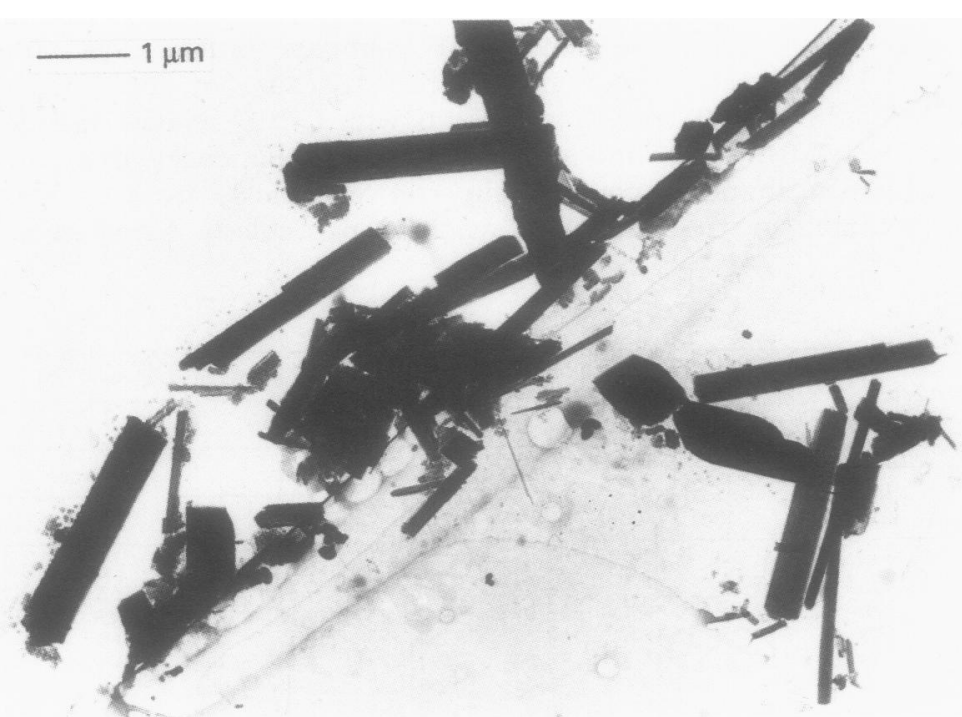

Figure 2 Electron micrograph of calcium pyrophosphate dihydrate (CPPD) crystals extracted from the interval synovial fluid sample from patient 6. Total yield of crystals $30 \mu \mathrm{g}$ : a mixture of M-CPPD (mean length $3.8 \mu \mathrm{m}$ ) and T-CPPD (rhomboid/cuboid shaped). case there was evidence of much more severe inflammation in the acute than in the interval sample.

Our results clearly show that in the acute episode samples there was a greater crystal load, larger crystals, and a greater ratio of monoclinic to triclinic CPPD crystals. Although the number of patients was relatively small, the consistency of the data, which show the same trend in each patient, and the size of the differences noted between the two samples, are impressive. Furthermore, the HRTEM and XRD analyses, both of which were carried out blind to the clinical data, and each of which used a quite different approach, showed a greater ratio of monoclinic to triclinic crystals in the acute episode SF compared with the interval fluid for every patient. The exact amount of monoclinic and triclinic material in each sample was difficult to compute. The data obtained by HRTEM are based on a morphological assessment of crystal habit. As discussed earlier, the XRD technique used is only semiquantitative; the relative intensities of the reflections for $\mathrm{M}$ - and T-CPPD are not related solely to the concentrations of each crystal form within a mixture. However, from measurements of the relative intensities of the key XRD reflections for $M$ - and T-CPPD, an indication of the relative abundance of the two forms was obtained. Changes in this ratio were observed in all nine patients, each acute sample having a greater ratio than the corresponding interval sample. This does not indicate that the concentration of M-CPPD was greater than that of T-CPPD in the acute SF sample, but rather that the ratio shifted in favour of M-CPPD. The morphology of a crystal does not always reflect its structure (although this is usually the case). This fact, together with the semiquantitative nature of XRD analysis, may explain the difference in monoclinic to triclinic ratios reported with each technique, those reported by HRTEM being greater than those recorded from XRD data. In contrast, with CPPD, there was no apparent correlation between the presence of small numbers of coexisting calcium HAP crystals and inflammation.

Previous data on the CPPD crystal loads in SF have also suggested the presence of a greater amount in pseudogout than in less inflammatory conditions. ${ }^{8}$ However, these data were based on cross sectional rather than longitudinal samples, and used chemical rather than gravimetric methods of analysis. The difference in methodology may explain the discrepancies in the total amount of mineral found. Each acute SF sample contained a greater concentration of CPPD, and a greater total crystal load, than the corresponding interval SF. However, the wide variation in concentration of SF CPPD associated with pseudogout suggests that there is not a clear threshold concentration for CPPD above which an inflammatory response is triggered. A threshold concentration might be associated with some other feature of the crystal population. Crystal habit (monoclinic versus triclinic) or particle size may be significant. ${ }^{11}$ 

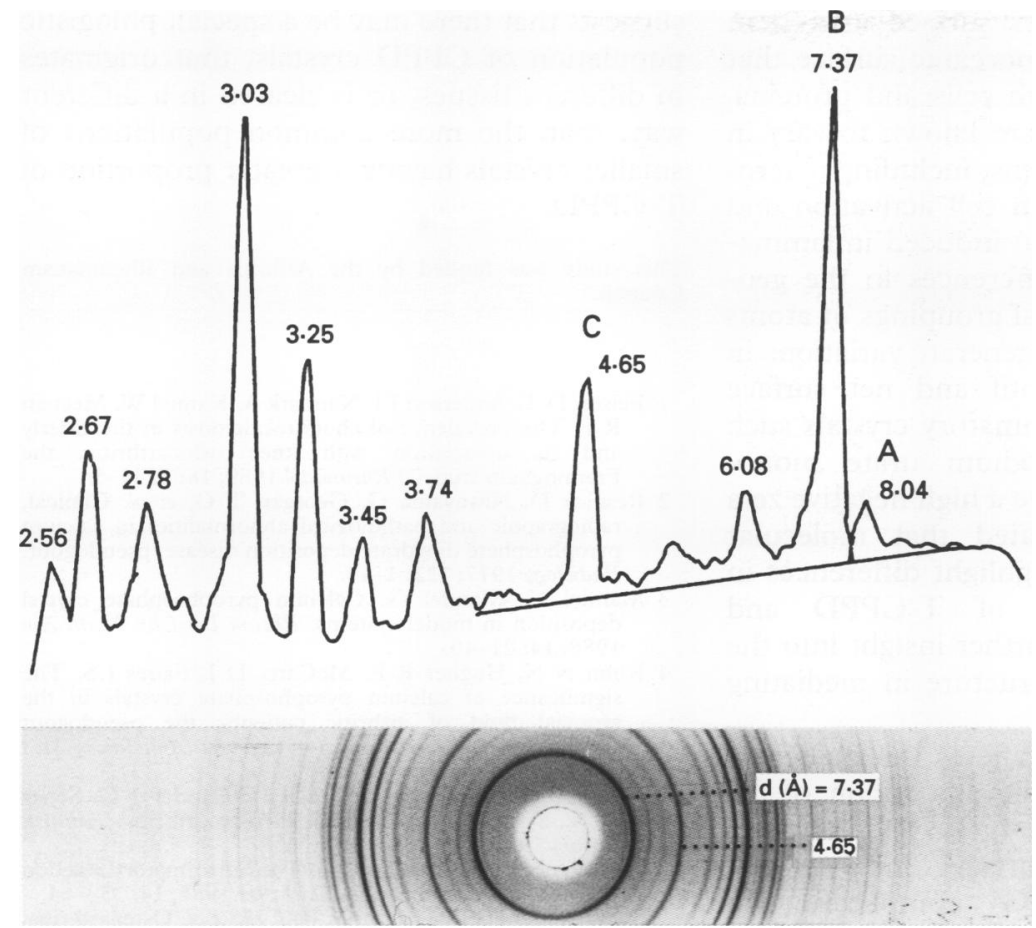

Figure 3 Diffraction pattern (below), and densitometric trace of $\mathrm{x}$ ray powder diffraction film, of extracted calcium pyrophosphate dihydrate (CPPD) crystals from the acute synovial fluid sample from patient 6: d-spacings (in angströms $(A)$ ) of principal reflections for $T$-CPPD and $M-C P P D$. Estimates of the ratio of $M-$ to $T-C P P D$ were obtained from the ratios of peak height ( $\mathrm{mm}$ ) of $B: A$, and of $C: A$. Reflections indicate that this acute sample consists of predominantly M-CPPD crystals.

The data reported here, showing a greater ratio of monoclinic to triclinic CPPD crystals in acute than in less inflammatory samples, and a greater mean crystal size, are both entirely new findings. These data raise two questions: first, why is there a difference in the type of crystal seen in the two samples, and second, does the crystal type dictate the synovial inflammatory response?

The crystals found in pseudogout are believed to arise through crystal shedding from a preformed deposit in cartilage; data to support this include the initiation of pseudogout attacks after solubilisation of deposits
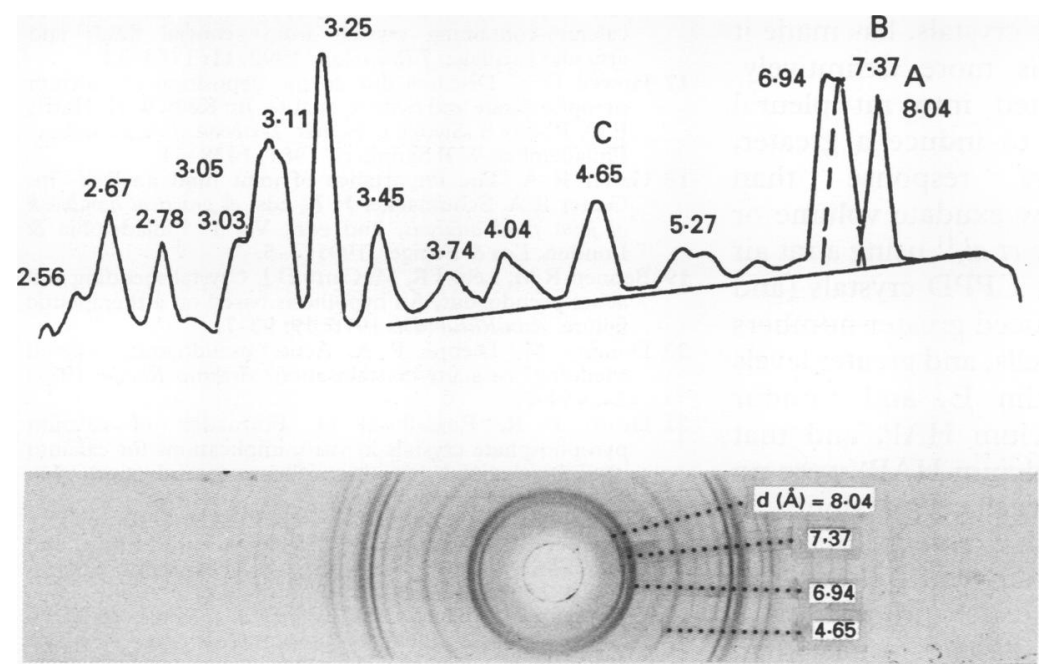

Figure 4 Diffraction pattern (below), and densitometric trace of $\mathrm{x}$ ray powder diffraction $X R D$ film, of extracted calcium pyrophosphate dihydrate (CPPD) crystals from the interval synovial fluid sample from patient 6: d-spacings (in ingströms $(A)$ ) of principal reflections for $T-C P P D$ and $M-C P P D$. Estimates of the ratio of $M-$ to $T-C P P D$ were obtained from the ratios of peak height $(\mathrm{mm})$ of $B: A$, and of $C: A$. Reflections indicate that this sample consists of a mixture of $M-C P P D$ and T-CPPD crystals. with magnesium lavage, ${ }^{19}$ and the disappearance of radiographic deposits during acute attacks. ${ }^{20}$ Our results indicated that larger crystals were associated with inflammatory SF. Phlogistic potential is related to the crystal surface available for interaction with cell membranes or proteins, however, and smaller crystals provide a greater specific surface area than large ones; in addition, their size may facilitate phagocytosis more readily, while some crystals may be too large to be phagocytosed. The evidence in the literature indicates that small CPPD crystals are more inflammatory, ${ }^{9}$ though the lower limit of this association is not known. It is possible that the acute samples also contained a number of very small crystals, difficult to identify, but of importance in the inflammatory response. Careful examination of these extracted CPPD, by HRTEM using magnifications that would allow easy visualisation of crystals as small as $100 \mathrm{~nm}$, failed to reveal identifiable $M-C P P D$ crystals of length $<0.08 \mu \mathrm{m}$. The absence of such small crystals may be explained by the phenomenon of Ostwald ripening, in which smaller crystals are forming continuously in a supersaturated solution, and immediately dissolving, their substance 'adding to' already formed larger crystals; thus, the crystal population contains very few very small crystals.

It may be that acute attacks are related to the increased proportion of monoclinic to triclinic CPPD, also observed in inflammatory SF. Given the mechanism described above, our data could be explained in one of two ways. The monoclinic crystals found in pseudogout attacks may be removed more quickly than triclinic crystals. Alternatively, they may be derived from a different type of deposit than the triclinic crystals which predominated in the interval fluids. The possibility of differences in the origins of the crystals, which might also explain the observed size differences, is currently under investigation, by examination of deposits which are formed in different parts of the joint. The third possibility is that M-CPPD crystallises during acute inflammation, but the thermodynamics of aqueous CPPD formation in vitro make this very unlikely. ${ }^{21}$

In vitro, M-CPPD converts, over time, to $T$-CPPD, ${ }^{21}$ suggesting that it is more soluble. The reduction in the monoclinic to triclinic ratio and the overall decrease in mean crystal size in the interval samples may then reflect dissolution of the $M$-form, or phase transformation to the $T$-form as the acute episode subsides. If both $\mathrm{M}$ - and T-CPPD reach the SF from joint tissues in large numbers, thus precipitating an attack of pseudogout, the more soluble M-CPPD may dissolve or revert to T-CPPD by the time the attack subsides, though it is unlikely that this could happen in vivo in inflammatory $S F$.

Whatever the reasons for the observed shifts in the proportions of M-CPPD and T-CPPD, our results raise the possibility that monoclinic CPPD crystals are more inflammatory than triclinic CPPD. Certainly, weight for weight, 
M-CPPD have a greater surface area than T-CPPD, and it is the inorganic surface that mediates interactions with cells and proteins. Indeed, crystal surfaces are known to vary in their ability to bind proteins, including macromolecules associated with cell activation and other mediators of crystal induced inflammation. ${ }^{12} 1322$ Structural differences in the geometric and stereochemical groupings of atoms within the crystal lattice generate variations in the exposed atomic motif and net surface charge of crystals. Inflammatory crystals such as CPPD and monosodium urate monohydrate, for example, have a high negative zeta potential. It is anticipated that molecular modelling studies will highlight differences in the surface chemistry of T-CPPD and $M-C P P D$ and provide further insight into the importance of surface structure in mediating pseudogout.

Another factor which affects the phlogistic potential of crystals is the adsorption of SF proteins onto surfaces. IgG, for example, adsorbs onto crystal surfaces in a charge mediated process; IgG complexes are positively charged under physiological conditions, and readily adsorb onto negatively charged surfaces. The adsorption mechanism is such that the Fc portion of the IgG molecule remains exposed and available for recognition by $\mathrm{Fc}$ receptors on PMN cells. ${ }^{12}$ It may be argued, therefore, that crystals with a net negative surface charge have a greater affinity for IgG and are more inflammatory. Sequential binding of other SF proteins may limit the interaction of those species capable of inducing an inflammatory response. ${ }^{23}$ Equally, the adsorption of organic material in such a manner as to limit the exposure of putative recognition sites would also affect this process. This surface binding may be different for different crystal forms.

There exist some in vitro data to support the theory that monoclinic CPPD is more inflammatory, ${ }^{10}{ }^{11}$ though the difficulties experienced in preparing pure monoclinic or triclinic crystals of the same crystal size, and of a size similar to that of biogenic crystals, has made it difficult to establish this more definitively. M-CPPD crystals injected into rat pleural cavity have been found to induce a greater, sustained inflammatory response than T-CPPD, as measured by exudate volume or PMN counts. ${ }^{10}$ Watanabe et $a l,{ }^{11}$ using a rat air pouch model, found that CPPD crystals (and especially M-CPPD) induced greater numbers of pouch exudate PMN cells, and greater levels of protease, prostaglandin $\mathrm{E}_{2}$ and tumour necrosis factor than calcium HAP, and that mixed crystals (CPPD/calcium HAP) were no more inflammatory than each crystal alone.

In conclusion, we have shown that the CPPD crystals associated with attacks of pseudogout include a greater proportion of monoclinic crystals, and larger crystals, than are seen in less inflammatory conditions. This suggests that there may be a special, phlogistic population of CPPD crystals, that originates in different tissues, or is cleared in a different way, than the more common populations of smaller crystals having a greater proportion of T-CPPD.

This study was funded by the Arthritis and Rheumatism Council.

1 Felson D T, Anderson J J, Naimark A, Kannel W, Meenan R F. The prevalence of chondrocalcinosis in the elderly and its association with knee osteoarthritis; the Framingham study. F Rheumatol 1989; 16: 1241-5.

2 Resnick D, Niwayama G, Georgan T G, et al. Clinical, radiographic and pathological abnormalities in calcium pyrophosphate dihydrate deposition disease: pseudogout. pyrophosphate dihydrate dep

3 Mandel N, Mandel G. Calcium pyrophosphate crystal deposition in model systems. Rheum Dis Clin North Am $1988 ; 14: 21-40$

4 Kohn N N, Hughes R E, McCarty D J, Faires J S. The significance of calcium pyrophosphate crystals in the synovial fluid of arthritic patients; the pseudogout syndrome. 11. Identification of crystals. Ann Intern Med 1962; 56: 738-45.

5 Gibilisco P A, Schumacher H R, Hollander J L, Soper $\mathrm{K}$ A. Synovial fluid crystals in osteoarthritis. Arthritis Rheum 1985; 28: 511-5.

6 Terkeltaub R A, Ginsberg M H. The inflammatory reaction to crystals. Rheum Dis Clin North Am 1988; 14: 353-64.

7 Schumacher H R, Gordon G, Paul H, et al. Osteoarthritis, crystal deposition and inflammation. Semin arthritis Rheum 1981; 11: 116-9.

8 Halverson P B, McCarty D J. Identification of hydroxyapatite crystals in synovial fluid. Arthritis Rheum 1979; 22 389-95.

9 Schumacher H R, Fishbein P, Phelps P, Tse R, Krauser R. Comparison of sodium urate and calcium pyrophosphate crystal size and other factors. Arthritis Rheum 1975; (suppl) 18: 783-93.

10 Roch-Arveiller $\mathrm{M}$, Legros $\mathrm{R}$, Chanaud $\mathrm{B}$, et al. Inflammatory reactions induced by various calcium pyrophosphate crystals. Biomed Pharmacother 1990; 44: pyrophosp

11 Watanabe W, Baker D G, Schumacher H R. Comparison of the acute inflammation induced by calcium pyrophosphate dihydrate, apatite and mixed crystals in the rat air pouch model of a synovial space. $f$ Rheumato 1992; 9: 1453-7.

12 Kozin F, McCarty D J. Protein binding to monosodium urate monohydrate, calcium pyrophosphate dihydrate and silicon dioxide crystals. 1. Physical characteristics. f Lab Clin Med 1977; 89: 1314-25.

13 Gordon T P, Ahern M J, Reid C, Roberts-Thompson P J. Studies on the interaction of rheumatoid factor with monosodium urate crystals and case report of coexistent tophaceous gout and rheumatoid arthritis. Ann Rheum Dis 1985; 44: 384-9.

14 Kellgren J H, Lawrence J S. Radiological assessment of Osteoarthritis. Ann Rheum Dis 1957; 16: 494-501.

15 McGill N W, Swan A J, Dieppe P A. Survival of calcium pyrophosphate dihydrate crystals in stored synovial fluids. Ann Rheum Dis 1991; 50: 939-41.

16 Swan A J, Heywood B R, Dieppe P A. Extraction of calcium-containing crystals from synovial fluids and articular cartilage. F Rheumatol 1992; 11: 1763-72.

17 Howell D S. Diseases due to the deposition of calcium pyrophosphate and hydroxyapatite. In: Kelly W N, Harris E D Ruddy S, Sledge C B, eds. Textbook of rheumatology. Philadelphia: W B Saunders, $1981 ; 1438-54$.

18 Gatter $R$ A. The importance of joint fluid analysis. In: Gatter R A, Schumacher H R, eds. A practical handbook of joint fluid analysis, 2nd edn, Vol 1. Philadelphia \& of joint fluid analysis, 2nd edn, Vol

19 Bennett R M, Lehr J R, McCarty D J. Crystal shedding and acute pseudogout. An hypothesis based on a therapeutic failure. Ann Rheum Dis 1976; 19: 93-7.

20 Doherty M, Dieppe P A. Acute pseudogout; "crystal shedding" or acute crystallisation? Arthritis Rheum 1981 24: 954-7.

21 Hearn $P$ R, Russell G G. Formation of calcium pyrophosphate crystals in vitro; implications for calcium phosphate crystal deposition disease (pseudogout). Ann Rheum Dis 1980; 39: 222-7.

22 Guerne P A, Terkeltaub R, Zuran B, Lotz M. Inflammatory microcrystals stimulate interleukin-6 production and secretion by human monocytes and synoviocytes. Arthritis Rheum 1989; 32: 1443-52.

23 Terkeltaub R, Curtiss L K, Tenner A J, Ginsberg M H. Lipoproteins containing apoprotein-B are a major crystals. F Clin Invest 1984; 73: 1719-30. 